\title{
For a brighter tomorrow
}

\author{
Photonics societies in the USA join forces in the National Photonics Initiative to increase photonics \\ research and development, grow the USA economy and improve national security.
}

On 23 May 2013, the American Physical Society, the IEEE Photonics Society, the Laser Institute of America, the Optical Society (OSA) and the International Society of Optics and Photonics (SPIE) launched the National Photonics Initiative (NPI) - a collaboration involving industry, academia and government.

The NPI was formed in response to a key recommendation of the National Academy of Sciences (NAS) 2012 report, "Optics and Photonics: Essential Technologies for our Nation," and seeks "to raise awareness of photonics and the impact of photonics on our everyday lives; increase cooperation and coordination among USA industry, government and academia to advance photonics-driven fields; and drive USA funding and investment in areas of photonics critical to maintaining US economic competitiveness and national security."

NPI's white paper "Lighting the Path to a Competitive, Secure Future," which had input from over 100 experts in industry, academia and government, identifies five key photonics-driven fields for guiding funding and investment - advanced manufacturing, communications and information technology, defence and national security, health and medicine, and energy.

"New opportunities arising from photonics, such as $3 \mathrm{D}$ printing, more efficient solar power, better nuclear threat identification, improved cancer detection and the bandwidth demands of a growing Internet, offer the potential for even greater societal impact in the next few decades. USA investment in photonics-driven fields will create jobs and grow the economy, protect and improve human life, and reposition the USA as a global technology leader," said Elizabeth Rogan, the CEO of OSA.

A major obstacle is the low recognition in the USA of the importance of photonics. "The EU, Germany, Korea, Taiwan and China all recognize the importance of photonics, and have taken actions," said Eugene Arthurs, the CEO of SPIE. "Right now, the USA, which has been a major contributor to the development of photonics, seems unable to recognize how much photonics enables today's technologies and to pull together its limited resources for best effect. We hope the NPI will better coordinate the fragmented effort in the USA and lead to better results

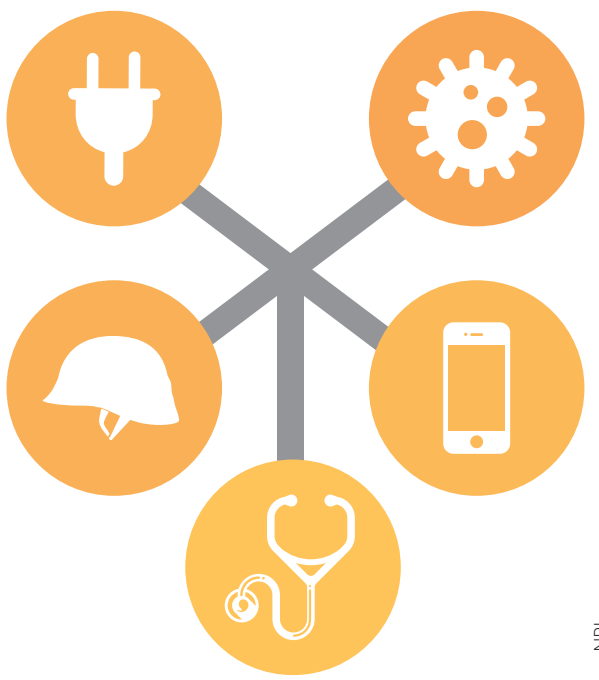

from the use of our resources. We also hope that by the photonics community better articulating the importance of photonics to the economy and society of the future we will unleash the potential of photonics in health, advanced manufacturing, energy and communications to continue the advances for people everywhere while driving down the cost curve. The challenge is to ensure adequate support to realize the opportunities," Arthurs emphasized.

The current federal budgetary constraints in the USA also don't help. "However, we feel that developing federal programs to encourage greater collaboration between industry, academia and government labs can be accomplished independent of increases in funding levels, which we also encourage," commented Richard Linke, the executive director of the IEEE Photonics Society.

For Rogan, another challenge is achieving measurable progress in an environment that doesn't have a culture of collaborating in an effective, precompetitive way. "There are several examples of efforts in Europe, such as Photonics21 and the Fraunhofer Model, that are effective vehicles working within their respective funding and culture climates. The beauty of an effort like this is that its impact will be global for the entire community."

Obviously, photonics societies in the USA will be critical in ensuring the success of the NPI. Rogan says that the OSA will communicate the importance of photonics to the general public and policymakers by galvanising its network of high-quality volunteer members and working with the other photonics societies to unite stakeholders in the five key areas.

SPIE seeks to promote the potential of photonics everywhere and recognizes that a worldwide effort is required if photonics is to address global challenges. "We need to pull together the strengths of academia, industry and government in the USA towards the goal of unleashing the power of photonics for the benefit of all," Arthurs stressed.

"The IEEE Photonics Society is happy to support NPI activities in the USA as well as in other countries where these activities may occur. We feel that the increased attention to the field will result in a better-informed

$\bar{z}$ public, a better-trained workforce and a more-productive industry," said Linke.

Certainly, the photonics community can be credited with major contributions that have improved the quality of our lives and promises continued advances with life-changing impact. However, this is not enough. "As much as our community understands the importance and amazing future potential of optics and photonics, we, as a community, need to do a better job at explaining what optics and photonics is and the many positive impacts it has and will continue to bring. For example, publishing this article gives us an opportunity to reach audiences that control funding, policies, strategic direction, research decisions and other areas critical to our future," said Rogan.

Arthurs concurs. "The community needs to better articulate the hows and whys and put more energy into communicating this, not to each other, but to the red and blue public, to budget makers in DC. That will be key to our continued success."

Linke also urges the lay community, which may be unfamiliar with the technology, to follow NPI activities and learn how the field has enabled technologies such as the Internet, ubiquitous cell phone use, lowcost international calls, financial networks that drive the world's economy, flat-screen televisions, LEDs for energy-efficient, longlife illumination, and medical diagnostics and treatment.

An initiative like this that involves both national and global cooperation will need concerted effort and persistence from each individual to make it successful. 